\title{
Leading the Change with Six Images of a Change Leader
}

\author{
Mojibola Bamidele-Sadiq, DBA student \\ m.sadiq.158@westcliff.edu
}

\begin{abstract}
Change is constant, and it is a continuous norm. It has even been said that, "to refuse to change is to be left behind" (anonymous). While difficult, this is still something that both individuals and organizations must cope with. The world is constantly changing, which explains why individuals and organizations that are open to change continue to survive. Many researchers have argued that an organization may only achieve a successful change when there is effective leadership. Effective leaders are those who understand when to change and how much to change. The purpose of this paper is to reiterate the importance of leadership in implementing a successful and transformational change in an organization. It will further explore a body of literature that supports and identifies roles leaders take on in the change management process.
\end{abstract}




\section{INTRODUCTION}

Today, organizations are constantly changing in their strategies, processes and procedures in response to the dynamism of business. Organizations are faced with internal and external environmental pressures, and in response to these pressures, they have to constantly change. Johansson and Heide (2008), explain that change is often driven by conditions in the surrounding environment and also triggered by needs within organizations. Elias and Mittal (2011), assert that virtually every organization will go through development and change at some point. While change revolves around both the organization and the employees, the employees are the main actors of change. Therefore, change is about the people in an organization. However, to lead these people, a change leader whose role is to act as the catalyst in the change process is essential. A change manager provides a structured approach to help people transition from a current state to a future state, as a result of change (Hoe, 2017).

More importantly, change cannot exist in a vacuum as it occurs between the organization and the people of the organization. Therefore, a successful change can be determined by identifying if the people were an integral part of the change process and throughout the process were building relationships and depending on team work. The personal leadership style of a change leader is the key to a transformational change taking place. Leaders must have confidence in themselves and their abilities so that those who look up to them as leaders will have confidence in them as well.

Hoogendoorn, Jonker, Schut and Treur (2007), explain that the need for a change may be prompted by an environment or by an organization. This paper analyses in detail why change is a survival strategy in today's business world, especially in the face of fierce competition. The methods used in this analysis are those of formalization, simulation, and verification. An analysis of the six images of a change manager in the pursuit of successful transformational changes will be included. Additionally, characteristics of leaders such as transparency, openness, trustworthiness, and honesty are found to be essential to achieve a desired change, and due to this, will be explored.

\section{The Six Images of a Change Manager}

Palmer, Dunford, and Buchanan (2016), identified the six images of a change manager as a director, interpreter, navigator, coach, caretaker and nurturer. A change leader must exhibit all six of these roles, which are not separate categories. Instead, they form two continua, from controlling to shaping management roles and implement outcomes that vary from planned to unintended. The boundaries of these six images are blurred, and their elements may overlap in practice (Palmer, Dunford, \& Buchanan, 2016). Although one or more of the six images may be more pronounced than the others, a transformational change leader is able to balance the mix.

In the role of a director, a change manager gives direction towards the expected outcome spelling out how the change would be achieved and providing the necessary resources required for the change. This image makes the change leader the grand commander in the change process. The one who designs the tactics and strategies that need to be adopted in order to achieve the desired result. The director maneuvers, manipulates, manages and monitors the teams' activities in the direction of the desired change (Palmer, Dunford, \& Buchanan, 2016). It is important for a change manager never to base actions on assumptions in the process of change. There 
must be a clear vision as well as a defined mission of what is to be achieved. This will guide the change. There must also be an understanding of what needs to change and the direction of the change in order for the leader to guide and control the activities of the team members appropriately.

The interpreter image requires more of a 'sense-making' approach involving 'sense breaking' and 'sense giving' to the situation. This simply means that the change must make sense to the people who will enact the change. In order to achieve this, a change leader must clearly communicate the purpose of the change in a manner that is easily understood by other team members. A change manager must know that everyone may not move at the same, thus, an assessment of the level of support from the team is necessary. Once a specified percentage of support is achieved, the late adopters will eventually catch up with the change (Palmer, Dunford, \& Buchanan, 2016). Whatever happens, it is the duty of change managers to offer interpretations of the change where they make sense to the people involved. In the opinion of Rouleau and Balogun (2010), sense-making is a social process of construction and reconstruction through which managers understand, interpret, and create sense for themselves and others of their changing organizational context and surroundings.

The image of a change manager as a coach is important because the actions or inactions of a leader can lead to a change that cannot be sustained or that may fail outright. Change can fail if the people enacting the change feel demotivated, if the vision is distorted, if people do not buy into the change, if there are dishonest leaders, or if organization politics and power are perceived. People tend to resist change either actively or passively, although, it is mostly passive as they would not want to be seen as opposing the organization or change outright (Palmer, Dunford, \& Buchanan, 2016). People will however actively resist change when it is perceived as likely to cause discomfort or is perceived as a threat. Lack of communication, perception of wrong timing for the change, lack of clarity, a negative legacy of past experiences, unclear advantages, dislike for change, strong attachment to status quo or organization culture, and a lack of or low tolerance for uncertainties are some of the reasons people resist change (Palmer, Dunford, \& Buchanan, 2016). As a navigator, a change leader must be able to manage through resistance, addressing the concerns of the team members while making them focus on the overall outcome which the change process aims to achieve. Change leaders must ensure that they always communicate effectively during the process of change management, perpetually assessing people's support of the change and projecting the change without threat in order to minimize resistance. Resistance to change can be managed using a contingency approach, although there is no specific best way of managing change (Urquhart \& Allison, 2014). A change manager needs to understand that change must take its natural course, and as such, people are bound to deny, resist, explore and finally commit to the new course. This is what Kubler-Ross (1969) identifies as the coping cycle is later described as the classic change curve (Schneider \& Goldwasser, 1998).

The change curve is a change management design model that describes the path the people and the organization follow in order to achieve the change result. It is a model that describes the four phases that people go through as they adjust to change. The phases are known as the introduction phase, the disruption phase (also known as the despair, resistance or apathy phase), the decision phase (also known as the make or break point) and lastly, the phase of embracing and 
committing to the change. It is worthy to note that although the phases are predictable, strong leadership is needed to know what to do at the point of resistance, despair or apathy. The role of a nurturer on behalf of the leader is necessary in order to achieve stability (Palmer, Dunford \& Buchanan, 2016).

Throughout the process of change, each situation will be different, and as such, a leader must determine the best approach as they progress (Burnes, 1996). This is where the role of the change leader as the caretaker comes into play. In this role, the leader must first ensure that resources are available in terms of capital and intellectual ability. Next, they must ensure process continuity such that no one's absence can halt or disrupt the process. The third step is to shape the process while understanding the direction of change in order to identify promptly when there may be some form of derailment. If this occurs, a change leader must develop an adequate plan to put things back on track. Lastly, a caretaker is to welcome constructive criticism. The failure of a change does not always indicate failure, but rather it serves as a learning tool to identify other better ways of achieving the desired result (Palmer, Dunford, \& Buchanan, 2016).

There are identified change management competencies required for effective leadership. The Change Management Institute (CMI, 2012) proposed a competency model which encompasses all the areas that require the attention of a change manager. The model identifies twelve skill areas of a transformational change leader and emphasizes a more strategic perspective of the change manager's ability to understand the environment, the stakeholders, and the sustainable outcomes.

Other inherent factors that may affect sustainable change and which a change manager must be mindful of are organizational culture, politics and power. If an organization's culture does not embrace change, a change manager will have to utilize more energy and resources in order to achieve buy-in. A change manager cannot be ignorant or neglect organizational politics. Instead of pretending that they do not exist, a change manager must engage in order to gain the desired political support necessary to achieve the change. In the opinion of Buchanan, Claydon, and Doyle (1999), organization politics play a major role in change implementation and this is why it must not be ignored, but rather managed in a change process. A change manager must know that some individuals are powerful in the organization. These individuals can either make or break a change program, and as such, change managers must strive to gain the buyin of such people in the organization. In the opinion of Mark and Wim (2008), the success of strategic change will depend not only on an organization's ability to implement new structures and processes, but also on the organization's ability to convey the new mission and priorities to its many stakeholders. In other words, communication is key in achieving successful organizational change.

\section{CONCLUSIONS}

The practical implication of analysis done in this paper is to identify the strategic roles that leaders play in the change management process while contributing to the body of literature regarding the strategic change management process. It is very important that change managers understand the change management process itself, the changes by understanding the vision, and the need for change. They can then create a shared understanding of the change with their team through effective and honest communication, a well-defined strategy and properly mapped out plans for the change. A change manager must know that change can fail and must be 
prepared for unplanned or unprecedented outcomes.

The further implication of this paper is the need for a strategic approach to managing change because change itself is a risky venture characterized by so many uncertainties. A change leader must be prepared to navigate through the process, weathering the storm until normalcy and the desired results are achieved. The likelihood of unintended outcomes is one of the major reasons why people tend to resist change. Unfortunately, change is inevitable but resistance to change is not inevitable. Therefore, change leaders must ensure to communicate effectively during the change management process, assessing people's support of the change and projecting the change without threat in order to minimize resistance. 


\section{REFERENCES}

Buchanan, D., Claydon, T., \& Doyle, M. (1999). Organization development and change: The legacy of the nineties. Human Resource Management Journal, 9(2), 20-37. Retrieved from https://search. proquest.com/docview/199387134?accountid=158986.

Burnes, B. (1996). Managing change: A strategic approach to organizational dynamics ( $2^{\text {nd }}$ ed.). London: Pitman.

Change management survey 2012. (2012). In Change Management Institute. Retrieved from http://www.changemanagement-institute.com/sites/default/files/cmiresearchcmi_survey2012finalreport.pdf

Elias, S. M., \& Mittal, R. (2011). The importance of supervisor support for a change initiative. International Journal of Organizational Analysis, 19(4), 305-316. http://dx.doi.org/10.1108/19348831111173432.

Hoe, S. L. (2017). What makes a competent change manager? The importance of developing the "right" attitudes. Development and Learning in Organizations, 31(5), 14-16. Retrieved from https://search. proquest.com/docview/1938399022?accountid=158986

Hoogendoorn, M., Jonker, C. M., Schut, M. C., \& Treur, J. (2007). Modeling centralized organization of organizational change. Computational and Mathematical Organization Theory, 13(2), 147-184. http://dx.doi.org/10.1007/s10588-006-9004-5

Johansson, C., \& Heide, M. (2008). Speaking of change: Three communication approaches in studies of organizational change. Corporate Communications, 13(3), 288-305. http://dx.doi.org/10.1108/13563280810893661.

Mark, V. V., \& Wim J.L. (2008). Communication, sensemaking and change as a chord of three strands. Corporate Communications, 13(3), 349-359. http://dx.doi.org/10.1108/13563280810893706

Palmer, I., Dunford, R., \& Buchanan, D. (2016). Managing organizational change: A multiple perspective approach (3rd ed.). New York: McGraw Hill.

Rouleau, L. \& Balogun, J. (2011). Middle managers, strategic sensemaking, and discursive competence. Journal of Management Studies, 48(5) 953-983. http://dx.doi.org/10.1111/ j.1467-6486.2010.00941.x

Urquhart, R., \& Allison, I. K. (2014). Towards a change leadership framework: Assessing capabilities within an IT service organization. Communications of the IIMA, 14(1), 87-103. Retrieved from https://search.proquest.com/docview/1765134458?accountid=158986 